\title{
Univoque numbers and an avatar of Thue-Morse
}

\author{
by \\ Jean-Paul Allouche (Orsay) and Christiane Frougny (Paris)
}

1. Introduction. Komornik and Loreti determined in [17] the smallest univoque real number in the interval $(1,2)$, i.e., the smallest number $\lambda \in$ $(1,2)$ such that 1 has a unique expansion $1=\sum_{j \geq 0} a_{j} / \lambda^{j+1}$ with $a_{j} \in\{0,1\}$ for every $j \geq 0$. The word "univoque" in this context seems to have been introduced (with a slightly different meaning) by Daróczy and Kátai in $[12,13]$, while unique expansions of the real number 1 were characterized by Erdős, Joó, and Komornik in [14]. The first author and Cosnard showed in [4] how the result of [17] parallels (and can be deduced from) their study of a certain set of binary sequences arising in the study of iterations of unimodal continuous functions on the unit interval (see $[11,2,1])$. The relevant sets of binary sequences occurring in $[2,1]$, resp. [17], can be defined by

$$
\begin{aligned}
\Gamma & :=\left\{A \in\{0,1\}^{\mathbb{N}}: \forall k \geq 0, \bar{A} \leq \sigma^{k} A \leq A\right\}, \\
\Gamma_{\text {strict }}: & =\left\{A \in\{0,1\}^{\mathbb{N}}: \forall k \geq 1, \bar{A}<\sigma^{k} A<A\right\},
\end{aligned}
$$

where $\sigma$ is the shift on sequences and the bar operation replaces 0 's by 1 's and 1's by 0's, i.e., if $A=\left(A_{n}\right)_{n \geq 0}$, then $\sigma A:=\left(a_{n+1}\right)_{n \geq 0}$ and $\bar{A}:=\left(1-a_{n}\right)_{n \geq 0}$; furthermore, $\leq$ denotes the lexicographical order on sequences induced by $0<1$, the notation $A<B$ meaning as usual that $A \leq B$ and $A \neq B$. The smallest univoque number in $(1,2)$ and the smallest nonperiodic sequence in $\Gamma$ both involve the Thue-Morse sequence (see for example [6] for more on this sequence).

It is tempting to generalize these sets to alphabets with more than two letters.

Definition 1. For $b$ a positive integer, we will say that the real number $\lambda>1$ is $\{0,1, \ldots, b\}$-univoque if the number 1 has a unique expansion as $1=\sum_{j \geq 0} a_{j} \lambda^{-(j+1)}$, where $a_{j} \in\{0,1, \ldots, b\}$ for all $j \geq 0$. Furthermore, if $\lambda>1$ is $\{0,1, \ldots,\lceil\lambda\rceil-1\}$-univoque, we will simply say that $\lambda$ is univoque.

2000 Mathematics Subject Classification: 11A63, 11B83, 11B85, 68R15, 11J81.

Key words and phrases: beta-expansion, univoque numbers, iteration of continuous functions, Thue-Morse sequence, uniform morphism, automatic sequence, transcendence. 
REMARK 1. If $\lambda>1$ is $\{0,1, \ldots, b\}$-univoque for some positive integer $b$, then $\lambda \leq b+1$. Also note that any integer $q \geq 2$ is univoque, since there is exactly one expansion $1=\sum_{j \geq 0} a_{j} q^{-(j+1)}$ with $a_{j} \in\{0,1, \ldots, q-1\}$, namely $1=\sum_{j \geq 0}(q-1) q^{-(j+1)}$.

Komornik and Loreti studied in [18] the reals $\lambda \in(1, b+1]$ that are $\{0,1, \ldots, b\}$-univoque. For their study, they introduced admissible sequences on the alphabet $\{0,1, \ldots, b\}$. Denote, as above, by $\sigma$ the shift on sequences, and by bar the operation that replaces every $t \in\{0,1, \ldots, b\}$ by $b-t$, i.e., if $A=\left(a_{n}\right)_{n \geq 0}$, then $\bar{A}:=\left(b-a_{n}\right)_{n \geq 0}$. Also denote by $\leq$ the lexicographical order on sequences induced by the natural order on $\{0,1, \ldots, b\}$. Then a sequence $A=\left(a_{n}\right)_{n \geq 0}$ on $\{0,1, \ldots, b\}$ is admissible if

$$
\begin{array}{ll}
\forall k \geq 0 \text { such that } a_{k}<b, & \sigma^{k+1} A<A, \\
\forall k \geq 0 \text { such that } a_{k}>0, & \sigma^{k+1} A>\bar{A} .
\end{array}
$$

(Note that our notation is not exactly the notation of [18], since our sequences are indexed by $\mathbb{N}$ and not $\mathbb{N} \backslash\{0\}$.) These sequences have the following property: the map that associates with a real $\lambda \in(1, b+1]$ the sequence of coefficients $\left(a_{j}\right)_{j \geq 0} \in\{0,1, \ldots, b\}$ of the greedy (i.e., lexicographically largest) expansion of $1,1=\sum_{j \geq 0} a_{j} \lambda^{-(j+1)}$, is a bijection from the set of $\{0,1, \ldots, b\}$-univoque $\lambda$ 's to the set of admissible sequences on $\{0,1, \ldots, b\}$ (see $[18])$.

Now there are two possible generalizations of the result of [17] on the smallest univoque number in $(1,2)$, i.e., the smallest admissible binary sequence. One is to look at the smallest (if any) admissible sequence on the alphabet $\{0,1, \ldots, b\}$, as did Komornik and Loreti in [18], the other is to look at the smallest (if any) univoque number in $(b, b+1)$, as did de Vries and Komornik in [22].

It so happens that the first author has already studied a generalization of the set $\Gamma$ to the case of more than two letters (see [1, Part 3]). Interestingly enough, unlike the study of $\Gamma$, this study was unrelated to iterations of continuous functions, being just a tempting formal arithmetico-combinatorial generalization of the study of the set $\Gamma$ of binary sequences to a similar set of sequences with more than two values.

The purpose of the present paper is threefold:

(1) to show how the 1983 study [1, Part 3, pp. 63-90] gives both the result of Komornik and Loreti in [18] on the smallest admissible sequence on $\{0,1, \ldots, b\}$, and the result of de Vries and Komornik in [22] on the smallest univoque number $\lambda \in(b, b+1)$ where $b$ is any positive integer;

(2) to bring to light a universal morphism that governs the smallest elements in (1) above, and to show that the infinite sequence generated by this morphism is an avatar of the Thue-Morse sequence; 
(3) to prove that the smallest univoque number in $(b, b+1)$ (where $b$ is any positive integer) is transcendental.

The paper consists of five sections. In Section 2 we recall some results of $[1$, Part 3, pp. 63-90] on the generalization of the set $\Gamma$ to a $(b+1)$-letter alphabet. Then we give some properties of the lexicographically least nonperiodic sequence of this set, completing the results of [1, Part 3, pp. 63-90]. In Section 3 we give two corollaries of the properties of this least sequence: one gives the result in [18], the other gives the result in [22]. The transcendence results are proven in the last section.

\section{The generalized $\Gamma$ and $\Gamma_{\text {strict }}$ sets}

Definition 2. Let $b$ be a positive integer, and $\mathcal{A}$ be a finite ordered set with $b+1$ elements $\alpha_{0}<\alpha_{1}<\cdots<\alpha_{b}$. The bar operation is defined on $\mathcal{A}$ by $\bar{\alpha}_{j}=\alpha_{b-j}$. We extend this operation to $\mathcal{A}^{\mathbb{N}}$ by ${\overline{\left(a_{n}\right)}}_{n \geq 0}:=\left(\bar{a}_{n}\right)_{n \geq 0}$. Let $\sigma$ be the shift on $\mathcal{A}^{\mathbb{N}}$, defined by $\sigma\left(\left(a_{n}\right)_{n \geq 0}\right):=\left(a_{n+1}\right)_{n \geq 0}$.

We define

$$
\begin{aligned}
\Gamma(\mathcal{A}) & :=\left\{A=\left(a_{n}\right)_{n \geq 0} \in \mathcal{A}^{\mathbb{N}}: a_{0}=\max \mathcal{A}, \forall k \geq 0, \bar{A} \leq \sigma^{k} A \leq A\right\}, \\
\Gamma_{\text {strict }}(\mathcal{A}) & :=\left\{A=\left(a_{n}\right)_{n \geq 0} \in \mathcal{A}^{\mathbb{N}}: a_{0}=\max \mathcal{A}, \forall k \geq 1, \bar{A}<\sigma^{k} A<A\right\} .
\end{aligned}
$$

REMARK 2. The set $\Gamma(\mathcal{A})$ was introduced by the first author in $[1$, Part 3, p. 63]. Note that there is a misprint in the definition on p. 66 in [1]: $a_{\beta-i}$ should be changed into $a_{\beta-1-i}$ as confirmed by the rest of the text.

REMARK 3. A sequence belongs to $\Gamma_{\text {strict }}(\mathcal{A})$ if and only if it belongs to $\Gamma(\mathcal{A})$ and is nonperiodic. Indeed, $\sigma^{k} A=A$ if and only if $A$ is $k$-periodic; if $\sigma^{k} A=\bar{A}$, then $\sigma^{2 k} A=A$, and the sequence $A$ is $2 k$-periodic.

REMARK 4. If the set $\mathcal{A}:=\{i, i+1, \ldots, i+z\}$, where $i$ and $z$ are integers, is equipped with the natural order, then for any $x \in \mathcal{A}$, we have $\bar{x}=2 i+z-x$. Indeed, following Definition 2 above, we write $\alpha_{0}:=i, \alpha_{1}:=i+1, \ldots, \alpha_{z}:=$ $i+z$. Hence, for any $j \in[0, z]$, we have $\bar{\alpha}_{j}=\alpha_{z-j}$, which can be rewritten $\overline{i+j}=i+z-j$, i.e., for any $x$ in $\mathcal{A}$, we have $\bar{x}=i+z-(x-i)=2 i+z-x$.

A first result is that the sets $\Gamma_{\text {strict }}(\mathcal{A})$ are closely linked to the set of admissible sequences whose definition was recalled in the introduction.

Proposition 1. Let $A=\left(a_{n}\right)_{n \geq 0}$ be a sequence in $\{0,1, \ldots, b\}^{\mathbb{N}}$ such that $a_{0}=t \in[0, b]$ and $A \neq b \quad b \quad b \quad \ldots$ Then $A$ is admissible if and only if $2 t>b$ and $A \in \Gamma_{\text {strict }}(\{b-t, b-t+1, \ldots, t\})$. (The order on $\{b-t, b-t+1$, $\ldots, t\}$ is induced by the order on $\mathbb{N}$. From Remark 4 the bar operation is given by $\bar{j}=b-j$.) 
Proof. First suppose that $2 t>b$ and $A \in \Gamma_{\text {strict }}(\{b-t, b-t+1, \ldots, t\})$. Then, for all $k \geq 1, \bar{A}<\sigma^{k} A<A$, which clearly implies that $A$ is admissible.

Conversely, suppose that $A$ is admissible. We thus have

$$
\begin{array}{ll}
\forall k \geq 1 \text { such that } a_{k-1}<b, & \sigma^{k} A<A, \\
\forall k \geq 1 \text { such that } a_{k-1}>0, & \sigma^{k} A>\bar{A} .
\end{array}
$$

We first prove that if $A$ is not a constant sequence, then

$$
\forall k \geq 1, \quad \bar{A}<\sigma^{k} A<A .
$$

We only prove that $\sigma^{k} A<A$; the remaining inequalities are proved in a similar way. If $a_{k-1}<b$, then $\sigma^{k} A<A$. If $a_{k-1}=b$, there are two cases: either

- $a_{0}=a_{1}=\cdots=a_{k-1}=b$; then if $a_{k}<b$ we clearly have $\sigma^{k} A<A$; if $a_{k}=b$, then the sequence $\sigma^{k} A$ begins with some block of $b$ 's followed by a letter $<b$, thus it begins with a block of $b$ 's shorter than the initial block of $b$ 's in $A$, hence $\sigma^{k} A<A$; or

- there exists an index $\ell$ with $1<\ell<k$ such that $a_{\ell-1}<b$ and $a_{\ell}=$ $a_{\ell+1}=\cdots=a_{k-1}=b$. As $A$ is admissible, we have $\sigma^{\ell} A<A$. It thus suffices to prove that $\sigma^{k} A \leq \sigma^{\ell} A$. This is clearly the case if $a_{k}<b$. On the other hand, if $a_{k}=b$, the sequence $\sigma^{k} A$ begins with a block of $b$ 's which is shorter than the initial block of $b$ 's in $\sigma^{\ell} A$, hence $\sigma^{k} A \leq \sigma^{\ell} A$.

Now, since $a_{0}=t$ and $\sigma^{k} A<A$ for all $k \geq 1$, we have $a_{k} \leq t$ for all $k \geq 0$. Similarly, since $\sigma^{k} A>\bar{A}$ for all $k \geq 1$, we have $a_{k} \geq b-t$ for all $k \geq 1$. Finally, $A>\bar{A}$ implies that $t=a_{0} \geq b-t$. Thus $2 t \geq b$ and $A \in \Gamma(\{b-t, b-t+1, \ldots, t\})$. Now, if $b=2 t$, then $\{b-t, b-t+1, \ldots, t\}=\{t\}$ and $\bar{t}=t$. This implies that $A=t t t \ldots$, which is not an admissible sequence.

REMARK 5. For $b=1$, this (easy) result is given without proof in [14] and proved in [4].

We need another definition from [1].

Definition 3. Let $b$ be a positive integer, and $\mathcal{A}$ be a finite ordered set with $b+1$ elements $\alpha_{0}<\alpha_{1}<\cdots<\alpha_{b}$. We suppose that $\mathcal{A}$ is equipped with a bar operation as in Definition 2. Let $A=\left(a_{n}\right)_{n \geq 0}$ be a periodic sequence of smallest period $T$, and with $a_{T-1}<\max \mathcal{A}$. Let $a_{T-1}=\alpha_{j}$ (thus $j<b$ ). Then $\Phi(A)$ is the $2 T$-periodic sequence beginning with $a_{0} a_{1} \ldots a_{T-2} \alpha_{j+1} \bar{a}_{0} \bar{a}_{1} \ldots \bar{a}_{T-2} \alpha_{b-j-1}$, i.e.,

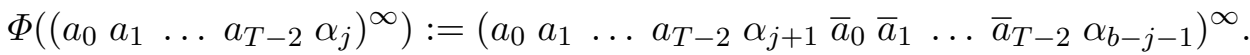

We first prove the following easy claim. 
Proposition 2. The smallest element of $\Gamma(\{b-t, b-t+1, \ldots, t\})$ (where $2 t>b)$ is the 2-periodic sequence $(t(b-t))^{\infty}=(t(b-t) t(b-t) t \ldots)$.

Proof. Since any sequence $A=\left(a_{n}\right)_{n \geq 0}$ in $\Gamma(\{b-t, b-t+1, \ldots, t\})$ begins in $t$, and satisfies $\sigma A \geq \bar{A}$, it must satisfy $a_{0}=t$ and $a_{1} \geq b-t$. Now if $a_{0}=t$ and $a_{1}=b-t$, then $A$ must be the 2-periodic sequence $(t(b-t))^{\infty}([1$, Lemma $2 \mathrm{~b}$, p. 73$])$. Since this periodic sequence trivially belongs to $\Gamma(\{b-t, b-t+1, \ldots, t\})$, it is its smallest element.

Denoting as usual by $\Phi^{s}$ the $s$ th iterate of $\Phi$, we state the following theorem which is a particular case of the theorem on pp. 72-73 of [1] about the smallest elements in certain subintervals of $\Gamma(\{0,1, \ldots, b\})$, and of the definition of $q$-mirror sequences given in [1, Section II, 1, p. 67] (here $q=2$ ).

Theorem 1 ([1]). Define $P:=(t(b-t))^{\infty}=(t(b-t) t(b-t) t \ldots)$. The smallest nonperiodic sequence in $\Gamma(\{b-t, b-t+1, \ldots, t\})$ (i.e., the smallest element of $\left.\Gamma_{\text {strict }}(\{b-t, b-t+1, \ldots, t\})\right)$ is the sequence

$$
M:=\lim _{s \rightarrow \infty} \Phi^{s}(P),
$$

that actually takes the (not necessarily distinct) values $b-t, b-t+1, t-1, t$. Furthermore, this sequence

$$
M=\left(m_{n}\right)_{n \geq 0}=t \quad b-t+1 \quad b-t \quad t \quad b-t \quad t-1 \quad \ldots
$$

can be recursively defined by

$$
\begin{aligned}
& \forall k \geq 0, \quad m_{2^{2 k}-1}=t, \\
& \forall k \geq 0, \quad m_{2^{2 k+1}-1}=b+1-t, \\
& \forall k \geq 0, \forall j \in\left[0,2^{k+1}-2\right], \quad m_{2^{k+1}+j}=\bar{m}_{j} .
\end{aligned}
$$

It was proven in [1] that the sequence $\lim _{s \rightarrow \infty} \Phi^{s}\left((t(b-t))^{\infty}\right.$ is 2automatic (for more about automatic sequences, see [7]). The second author noted that this sequence is actually a fixed point of a uniform morphism of length 2 as soon as the cardinality of the set $\{b-t, b-t+1, \ldots, b\}$ is at least 4 , i.e., $2 t \geq b+3$. (Recall that we always have $t \geq b-t$, i.e., $2 t \geq b$.) More precisely, we have Theorem 2 below, where the Thue-Morse sequence pops up, as in [1] and in [18], but also as in [2] and [17]. Before stating this theorem we give a definition. by

Definition 4. The "universal" morphism $\Theta$ is defined on $\left\{e_{0}, e_{1}, e_{2}, e_{3}\right\}$

$$
\Theta\left(e_{3}\right):=e_{3} e_{1}, \quad \Theta\left(e_{2}\right):=e_{3} e_{0}, \quad \Theta\left(e_{1}\right):=e_{0} e_{3}, \quad \Theta\left(e_{0}\right):=e_{0} e_{2} .
$$

Note that this morphism has an infinite fixed point beginning in $e_{3}$,

$$
\Theta^{\infty}\left(e_{3}\right)=\lim _{k \rightarrow \infty} \Theta^{k}\left(e_{3}\right)=e_{3} e_{1} e_{0} e_{3} e_{0} e_{2} e_{3} e_{1} e_{0} e_{2} \ldots
$$


TheOREM 2. Let $\left(\varepsilon_{n}\right)_{n \geq 0}$ be the Thue-Morse sequence defined by $\varepsilon_{0}=0$ and $\varepsilon_{2 k}=\varepsilon_{k}$ and $\varepsilon_{2 k+1}=1-\varepsilon_{k}$ for all $k \geq 0$. Then the smallest nonperiodic sequence $M=\left(m_{n}\right)_{n \geq 0}$ in $\Gamma(\{b-t, b-t+1, \ldots, t\})$ satisfies

$$
\forall n \geq 0, \quad m_{n}=\varepsilon_{n+1}-(2 t-b-1) \varepsilon_{n}+t-1 .
$$

Using the morphism $\Theta$ introduced in Definition 4 above we thus have:

- if $2 t \geq b+3$, then $M$ is the fixed point beginning in $t$ of the morphism deduced from $\Theta$ by renaming $e_{0}, e_{1}, e_{2}, e_{3}$ respectively $b-t, b-t+1$, $t-1, t$ (note that the condition $2 t \geq b+3$ implies that these four numbers are distinct);

- if $2 t=b+2$ (thus $b-t+1=t-1)$, then $M$ is the pointwise image of the fixed point beginning in $e_{3}$ of the morphism $\Theta$ under the map $g$ defined by $g\left(e_{3}\right):=t, g\left(e_{2}\right)=g\left(e_{1}\right):=t-1, g\left(e_{0}\right):=b-t$;

- if $2 t=b+1$ (thus $b-t=t-1$ and $b-t+1=t$ ), then $M$ is the pointwise image of the fixed point beginning in $e_{3}$ of the morphism $\Theta$ under the map $h$ defined by $h\left(e_{3}\right)=h\left(e_{1}\right):=t, h\left(e_{2}\right)=h\left(e_{0}\right):=t-1$.

Proof. Let us first prove that the sequence $M=\left(m_{n}\right)_{n \geq 0}$ is equal to the sequence $\left(u_{n}\right)_{n \geq 0}$, where $u_{n}:=\varepsilon_{n+1}-(2 t-b-1) \varepsilon_{n}+t-1$. It suffices to prove that $\left(u_{n}\right)_{n \geq 0}$ satisfies the recursive relations defining $\left(m_{n}\right)_{n \geq 0}$ that are given in Theorem 1 . Recall that $\varepsilon_{n}$ is equal to the parity of the sum of the binary digits of $n$ (see [6] for example). Hence, for all $k \geq 0, \varepsilon_{2^{2 k}-1}$ $=0, \varepsilon_{2^{2 k+1}-1}=1$, and $\varepsilon_{2^{2 k}}=\varepsilon_{2^{2 k+1}}=1$. This implies that for all $k \geq 0$, $u_{2^{2 k}-1}=t$ and $u_{2^{2 k+1}-1}=b+1-t$. Furthermore, for all $k \geq 0$ and $j \in$ $\left[0,2^{k+1}-2\right]$, we have $\varepsilon_{2^{k+1}+j}=1-\varepsilon_{j}$ and $\varepsilon_{2^{k+1}+j+1}=1-\varepsilon_{j+1}$. Hence $u_{2^{k+1}+j}=b-u_{j}=\bar{u}_{j}$.

To show how the "universal" morphism $\Theta$ enters the picture, we study the sequence $\left(v_{n}\right)_{n \geq 0}$ with values in $\{0,1\}^{2}$ defined by $v_{n}:=\left(\varepsilon_{n}, \varepsilon_{n+1}\right)$ for all $n \geq 0$. Since $v_{2 n}=\left(\varepsilon_{n}, 1-\varepsilon_{n}\right)$ and $v_{2 n+1}=\left(1-\varepsilon_{n}, \varepsilon_{n+1}\right)$ for all $n \geq 0$, we clearly have

$$
\begin{aligned}
& \text { if } v_{n}=(0,0) \text {, then } v_{2 n}=(0,1) \text { and } v_{2 n+1}=(1,0) \text {, } \\
& \text { if } v_{n}=(0,1) \text {, then } v_{2 n}=(0,1) \text { and } v_{2 n+1}=(1,1) \text {, } \\
& \text { if } v_{n}=(1,0) \text {, then } v_{2 n}=(1,0) \text { and } v_{2 n+1}=(0,0) \text {, } \\
& \text { if } v_{n}=(1,1) \text {, then } v_{2 n}=(1,0) \text { and } v_{2 n+1}=(0,1) \text {. }
\end{aligned}
$$

This exactly means that $\left(v_{n}\right)_{n \geq 0}$ is the fixed point beginning in $(0,1)$ of the 2-morphism

$$
\begin{aligned}
& (0,0) \rightarrow(0,1)(1,0), \\
& (0,1) \rightarrow(0,1)(1,1), \\
& (1,0) \rightarrow(1,0)(0,0), \\
& (1,1) \rightarrow(1,0)(0,1) .
\end{aligned}
$$


We may define $e_{0}:=(1,0), e_{1}:=(1,1), e_{2}:=(0,0), e_{3}:=(0,1)$. Then the above morphism can be written

$$
e_{3} \rightarrow e_{3} e_{1}, \quad e_{2} \rightarrow e_{3} e_{0}, \quad e_{1} \rightarrow e_{0} e_{3}, \quad e_{0} \rightarrow e_{0} e_{2},
$$

which is the morphism $\Theta$. The above construction shows that the sequence $\left(v_{n}\right)_{n \geq 0}$ is a fixed point of $\Theta$.

Now, define the map $\omega$ on $\{0,1\}^{2}$ by

$$
\omega((x, y)):=y-(2 t-b-1) x+t-1 .
$$

We have $\omega\left(v_{n}\right)=m_{n}$ for all $n \geq 0$. Thus

- if $2 t \geq b+3$, the sequence $\left(m_{n}\right)_{n \geq 0}$ takes exactly four distinct values, namely $b-t, b-t+1, t-1, t$. This implies that $\left(m_{n}\right)_{n \geq 0}$ is the fixed point beginning in $t$ of the morphism obtained from $\Theta$ by renaming the letters, i.e., $e_{3} \rightarrow t, e_{2} \rightarrow(t-1), e_{1} \rightarrow(b-t+1), e_{0} \rightarrow(b-t)$. The morphism can thus be written $t \rightarrow t(b-t+1),(t-1) \rightarrow t(b-t)$, $(b-t+1) \rightarrow(b-t) t,(b-t) \rightarrow(b-t)(t-1) ;$

- if $2 t=b+2($ resp. $2 t=b+1)$ the sequence $\left(m_{n}\right)_{n \geq 0}$ takes exactly three (resp. two) values, namely $b-t, t-1, t$ (resp. $t-1, t)$. It is still the pointwise image under $\Theta$ of the sequence $\left(v_{n}\right)_{n \geq 0}$. Renaming the fixed point of $\Theta$ under $g$ (resp. $h$ ) as in the statement of Theorem 2 only takes into account that the integers $b-t, b-t+1, t-1, t$ are not distinct.

REMARK 6. The reason for the choice of indices for $e_{3}, e_{2}, e_{1}, e_{0}$ is that the order of indices is the same as the natural order on the integers $t, t-1$, $b-t+1, b-t$ to which they correspond when $2 t \geq b+3$. In particular, if $b=t=3$, the morphism reads: $3 \rightarrow 31,2 \rightarrow 30,1 \rightarrow 03,0 \rightarrow 02$. Interestingly enough, though not surprisingly, this morphism also occurs (up to renaming the letters once more) in the study of infinite square-free sequences on a 3-letter alphabet. Namely, in [9], Berstel proves that the square-free Istrail sequence [15], originally defined (with no mention of the Thue-Morse sequence) as the fixed point of the (nonuniform) morphism $0 \rightarrow 12,1 \rightarrow 102,2 \rightarrow 0$, is actually the pointwise image of the fixed point beginning in 1 of a 2-morphism $\Theta^{\prime}$ on the 4-letter alphabet $\{0,1,2,3\}$ under the map $0 \rightarrow 0,1 \rightarrow 1,2 \rightarrow 2,3 \rightarrow 0$. The morphism $\Theta^{\prime}$ is given by

$$
\Theta^{\prime}(0)=12, \quad \Theta^{\prime}(1)=13, \quad \Theta^{\prime}(2)=20, \quad \Theta^{\prime}(3)=21 .
$$

The reader will note immediately that $\Theta^{\prime}$ is another avatar of $\Theta$ obtained by renaming letters as follows: $0 \rightarrow 2,1 \rightarrow 3,2 \rightarrow 0,3 \rightarrow 1$. This, in particular, shows that the sequence $\left(m_{n}\right)_{n \geq 0}$, in the case where $2 t=b+2$, is the fixed point of the nonuniform morphism $t \rightarrow t(t-1)(b-t),(t-1) \rightarrow t(b-t)$, $(b-t) \rightarrow(t-1)$, i.e., an avatar of Istrail's square-free sequence. Furthermore, it follows from [9] that this sequence on three letters cannot be the fixed point 
of a uniform morphism. A last remark is that the square-free Braunholtz sequence on three letters given in [10] (see also [9, p. 18-07]) is exactly our sequence $\left(m_{n}\right)_{n \geq 0}$ when $t=b=2$, i.e., the sequence $210201210120 \ldots$

\section{Small admissible sequences and small univoque numbers with given integer part}

3.1. Small admissible sequences with values in $\{0,1, \ldots, b\}$. In [18] the authors are interested in the smallest admissible sequence with values in $\{0,1, \ldots, b\}$, where $b$ is an integer $\geq 1$. They prove in particular the following result, which is an immediate corollary of our Theorem 2 .

COROLlary 1 (Theorems 4.3 and 5.1 of [18]). Let $b$ be an integer $\geq 1$. The smallest admissible sequence with values in $\{0,1, \ldots, b\}$ is the sequence $\left(z+\varepsilon_{n+1}\right)_{n \geq 0}$ if $b=2 z+1$, and $\left(z+\varepsilon_{n+1}-\varepsilon_{n}\right)_{n \geq 0}$ if $b=2 z$.

Proof. Let $A=\left(a_{n}\right)_{n \geq 0}$ be the smallest (nonconstant) admissible sequence with values in $\{0,1, \ldots, b\}$. Since $A>\bar{A}$, we must have $a_{0} \geq \bar{a}_{0}=$ $b-a_{0}$.

Thus, if $b=2 z+1$ we have $a_{0} \geq z+1$. We also have, for all $i \geq 0$, $\bar{a}_{0} \leq a_{i} \leq a_{0}$. Now the smallest element of $\Gamma(\{b-z-1, b-z, \ldots, z-1, z+1\})$ is the smallest admissible sequence on $\{0,1, \ldots, b\}$ that begins in $z+1$. Hence this is the smallest admissible sequence with values in $\{0,1, \ldots, b\}$. Theorem 2 shows that this sequence is $\left(m_{n}\right)_{n \geq 0}$ with $m_{n}=\varepsilon_{n+1}+z$ for all $n \geq 0$.

If $b=2 z$, we have $a_{0} \geq z$. But if $a_{0}=z$, then $\bar{a}_{0}=z$, and the condition of admissibility implies that $a_{n}=z$ for all $n \geq 0$ and $\left(a_{n}\right)_{n \geq 0}$ would be the constant sequence $(z z z \ldots)$. Hence we must have $a_{0} \geq z+1$. Now the smallest element of $\Gamma(\{b-z-1, b-z, \ldots, z-1, z+1\})$ is the smallest admissible sequence on $\{0,1, \ldots, b\}$ that begins in $z+1$. Hence this is the smallest admissible sequence with values in $\{0,1, \ldots, b\}$. Theorem 2 implies that this sequence is $\left(m_{n}\right)_{n \geq 0}$ with $m_{n}=\varepsilon_{n+1}-\varepsilon_{n}+z$ for all $n \geq 0$.

3.2. Small univoque numbers with given integer part. We are interested here in the univoque numbers $\lambda$ in an interval $(b, b+1]$ with $b$ a positive integer. This set was studied in [16], where it was proved to have Lebesgue measure 0 . Since $1=\sum_{j \geq 0} a_{j} \lambda^{-(j+1)}$ and $\lambda \in(b, b+1]$, and $a_{0} \leq b$, the fact that the expansion of 1 is unique, hence equal to the greedy expansion, implies that $a_{0}=b$. In other words, we study the admissible sequences with values in $\{0,1, \ldots, b\}$ that begin in $b$, i.e., the set $\Gamma_{\text {strict }}(\{0,1, \ldots, b\})$. A corollary of Theorem 2 is that, for any positive integer $b$, there exists a smallest univoque number belonging to $(b, b+1]$. This result was obtained in [22] (see the penultimate remark in that paper); it generalizes the result obtained for $b=1$ in [17]. 
COROLlary 2. For any positive integer $b$, there exists a smallest univoque number in $(b, b+1]$. It is the solution of the equation $1=\sum_{n \geq 0} d_{n} \lambda^{-n-1}$, where $d_{n}:=\varepsilon_{n+1}-(b-1) \varepsilon_{n}+b-1$ for all $n \geq 0$.

Proof. It suffices to apply Theorem 2 with $t=b$.

4. Transcendence results. We now prove, mimicking the proof given in [3], that numbers $\lambda$ such that the $\lambda$-expansion of 1 is given by the sequence $\left(m_{n}\right)_{n \geq 0}$ are transcendental. This generalizes the transcendence results of [3] and [18].

TheOREM 3. Let $b$ be an integer $\geq 1$ and $t \in[0, b]$ be an integer such that $2 t \geq b+1$. Define the sequence $\left(m_{n}\right)_{n \geq 0}$ as in Theorem 2 by $m_{n}$ $:=\varepsilon_{n+1}-(2 t-b-1) \varepsilon_{n}+t-1$ for all $n \geq 0$, thus $\left(m_{n}\right)_{n \geq 0}$ begins with $t b-t+1 b-t t b-t t-1 \ldots$ Then the number $\lambda \in(1, b+1)$ defined by $1=\sum_{n \geq 0} m_{n} \lambda^{-n-1}$ is transcendental.

Proof. Define the \pm 1 Thue-Morse sequence $\left(r_{n}\right)$ by $r_{n}:=(-1)^{\varepsilon_{n}}$. We clearly have $r_{n}=1-2 \varepsilon_{n}$ (recall that $\varepsilon_{n}$ is 0 or 1 ). It is also immediate that the function $F$ defined for the complex numbers $X$ with $|X|<1$ by $F(X)=\sum_{n \geq 0} r_{n} X^{n}$ satisfies $F(X)=\prod_{k \geq 0}\left(1-X^{2^{k}}\right)$ (see, e.g., [6]). Since

$$
2 m_{n}=2 \varepsilon_{n+1}-2(2 t-b-1) \varepsilon_{n}+2 t-2=b-r_{n+1}+(2 t-b-1) r_{n}
$$

we have, for $|X|<1$,

$$
2 X \sum_{n \geq 0} m_{n} X^{n}=((2 t-b-1) X-1) F(X)+1+\frac{b X}{1-X} .
$$

Taking $X=1 / \lambda$ where $1=\sum_{n \geq 0} m_{n} \lambda^{-n-1}$, we get the equation

$$
2=\left((2 t-b-1) \lambda^{-1}-1\right) F(1 / \lambda)+1+\frac{b}{\lambda-1} .
$$

Now, if $\lambda$ were algebraic, then this equation shows that $F(1 / \lambda)$ would be an algebraic number. But, since $1 / \lambda$ would then be an algebraic number in $(0,1)$, the quantity $F(1 / \lambda)$ would be transcendental from a result of Mahler [19], giving a contradiction.

REMARK 7 . In particular the $\{0,1, \ldots, b\}$-univoque number corresponding to the smallest admissible sequence with values in $\{0,1, \ldots, b\}$ is transcendental, as proved in [18] (Theorems 4.3 and 5.9). Also the smallest univoque number belonging to $(b, b+1)$ is transcendental.

5. Conclusion. There are many papers dealing with univoque numbers. We just mention here the study of univoque Pisot numbers. The authors together with K. G. Hare determined in [5] the smallest univoque Pisot number, which happens to have algebraic degree 14 . Note that the number 
corresponding to the sequence of Proposition 2 is the larger real root of the polynomial $X^{2}-t X-(b-t+1)$, hence a Pisot number (which is unitary if $t=b$ ). Also note that for any $b \geq 2$, the real number $\beta$ such that the $\beta$-expansion of 1 is $b 1^{\infty}$ is a univoque Pisot number in $(b, b+1)$. It would be interesting to determine the smallest univoque Pisot number in $(b, b+1)$ : the case $b=1$ was addressed in [5], but the proof uses heavily the fine structure of Pisot numbers in $(1,2)$ (see $[8,20,21])$. A similar study of Pisot numbers in $(b, b+1)$ would certainly help.

Acknowledgments. The authors thank M. de Vries and V. Komornik for their remarks on a previous version of this paper.

\section{References}

[1] J.-P. Allouche, Théorie des Nombres et Automates, Thèse d'État, Bordeaux, 1983, http://tel.archives-ouvertes.fr/tel-00343206/fr/.

[2] J.-P. Allouche et M. Cosnard, Itérations de fonctions unimodales et suites engendrées par automates, C. R. Acad. Sci. Paris Sér. I 296 (1983), 159-162.

[3] - , - The Komornik-Loreti constant is transcendental, Amer. Math. Monthly 107 (2000), 448-449.

[4] -, -, Non-integer bases, iteration of continuous real maps, and an arithmetic selfsimilar set, Acta Math. Hungar. 91 (2001), 325-332.

[5] J.-P. Allouche, Ch. Frougny and K. Hare, On univoque Pisot numbers, Math. Comp. 76 (2007), 1639-1660.

[6] J.-P. Allouche and J. Shallit, The ubiquitous Prouhet-Thue-Morse sequence, in: C. Ding et al. (eds.), Sequences and Their Applications (Singapore, 1998), Springer, 1999, 1-16.

[7] -, -, Automatic Sequences. Theory, Applications, Generalizations, Cambridge Univ. Press, Cambridge, 2003.

[8] M. Amara, Ensembles fermés de nombres algébriques, Ann. Sci. École Norm. Sup. 83 (1966), 215-270.

[9] J. Berstel, Sur la construction de mots sans carré, Sém. Théor. Nombres Bordeaux (1978-1979), Exp. 18, 18-01-18-15.

[10] C. H. Braunholtz, An infinite sequence on 3 symbols with no adjacent repeats (Solution to Problem 439 posed by H. Noland), Amer. Math. Monthly 70 (1963), 675-676.

[11] M. Cosnard, Étude de la classification topologique des fonctions unimodales, Ann. Inst. Fourier (Grenoble) 35 (1985), 59-77.

[12] Z. Daróczy and I. Kátai, Univoque sequences, Publ. Math. Debrecen 42 (1993), 397-407.

[13] —, -, On the structure of univoque numbers, ibid. 46 (1995), 385-408.

[14] P. Erdős, I. Joó and V. Komornik, Characterization of the unique expansions $1=$ $\sum_{i=1}^{\infty} q^{-n_{i}}$, and related problems, Bull. Soc. Math. France 118 (1990), 377-390.

[15] S. Istrail, On irreductible [sic] languages and nonrational numbers, Bull. Math. Soc. Sci. Math. R. S. Roumanie 21 (1977), 301-308.

[16] I. Kátai and G. Kallós, On the set for which 1 is univoque, Publ. Math. Debrecen 58 (2001), 743-750.

[17] V. Komornik and P. Loreti, Unique developments in non-integer bases, Amer. Math. Monthly 105 (1998), 636-639. 
[18] V. Komornik and P. Loreti, Subexpansions, superexpansions and uniqueness properties in non-integer bases, Period. Math. Hungar. 44 (2002), 197-218.

[19] K. Mahler, Arithmetische Eigenschaften der Lösungen einer Klasse von Funktionalgleichungen, Math. Ann. 101 (1929), 342-366; Corrigendum, ibid. 103 (1930), 532.

[20] F. L. Talmoudi, Sur les nombres de $S \cap[1,2]$, C. R. Acad. Sci. Paris Sér. Math. 285 (1977), 969-971.

[21] - Sur les nombres de $S \cap[1,2[$, ibid. 287 (1978), 739-741.

[22] M. de Vries and V. Komornik, Unique expansions of real numbers, preprint, 2007, http://arxiv.org/abs/math/0609708v3.

CNRS, LRI, UMR 8623

Université Paris-Sud, Bâtiment 490

F-91405 Orsay Cedex, France

E-mail: allouche@lri.fr
LIAFA, CNRS UMR 7089

Case 7014

F-75205 Paris Cedex 13, France

and

Université Paris 8

E-mail: Christiane.Frougny@liafa.jussieu.fr

Received on 9.11.2007

and in revised form on 5.10.2008 